\title{
Extensive sequencing of the cystic fibrosis transmembrane regulator gene: Assay validation and unexpected benefits of developing a comprehensive test
}

Charles M. Strom, MD, PhD, Donghui Huang, PhD, Christina Chen, Arlene Buller, PhD, Mei Peng, PhD, Franklin Quan, PhD, Joy Redman, MS, and Weimin Sun, MD, PhD

\begin{abstract}
Purpose: To develop a sequencing assay for the CFTR gene to identify mutations in patients with cystic fibrosis (CF). Methods: An automated assay format was developed to sequence all exons and splice junctional sequences, the promotor region, and parts of introns 11 and 19. Results: After validating the assay using 20 known samples, DNA of seven patients, four of whom were heterozygous for a known CF mutation, was sequenced. Known CF mutations were detected in seven of the eight chromosomes, and a novel missense mutation was detected in the eighth. In addition, this assay allowed 14 ambiguous results obtained using the Roche CF gold strips to be resolved. Three false-positive diagnoses were prevented; a different mutation at the same codon was identified in two patients and confirmation was provided in the remaining nine cases. Conclusions: Sequencing of the CFTR gene provides important information for CF patients and is a valuable adjunct to a carrier screening program to resolve ambiguities in panel testing. Genet Med 2003:5(1):9-14.
\end{abstract}

Key Words: cystic fibrosis, DNA sequencing, mutation detection

Cystic fibrosis (CF) is one of the most common recessive genetic diseases in North America. The disease is caused by mutations in the $\mathrm{CF}$ transmembrane regulator (CFTR) gene, which was cloned in $1989 . .^{1-3}$ Thus far more than 1,000 different CF mutations have been described. ${ }^{4}$ The American College of Medical Genetics (ACMG) has recommended a core panel of 25 mutations and 4 reflex polymorphisms for routine carrier screening for CF. ${ }^{5}$ The College panel is expected to identify approximately $97 \%$ of mutations in Ashkenazi Jews, $88 \%$ of mutations in non-Hispanic Caucasians, ${ }^{6}$ and much lower percentages in other ethnic groups. ${ }^{5-7}$ So-called extended panels do not increase these detection rates appreciably. ${ }^{5,6}$ Therefore, many CF patients will have one or both of their mutations unidentified using currently available screening techniques. These individuals, their parents, and extended family members can benefit from carrier detection and prenatal diagnosis if their rare or novel mutations are identified. Therefore, we developed an automated DNA sequence analysis-based assay capable of detecting all but 13 of the 1004 CF mutations described as of August 2002. ${ }^{4}$ As the incidence of these 13

From the Molecular Genetics Laboratory, Quest Diagnostics Nichols Institute, San Juan Capistrano, California.

Charles M. Strom, MD, PhD, Molecular Genetics Laboratory, Quest Diagnostics Nichols Institute, 33608 Ortega Highway, San Juan Capistrano, CA 92690.

Received: September 19, 2002.

Accepted: October 29, 2002.

DOI: 10.1097/01.GIM.0000046362.66762.E9 mutations is not known, it is impossible to calculate the percentage of CF chromosomes that will actually be identified by this assay.

This report describes the development and clinical validation of this assay and how the ability to selectively sequence any sequence containing an ACMG mutation has allowed us to resolve ambiguous results in our carrier screening program and avoid misdiagnoses.

\section{MATERIALS AND METHODS}

\section{Assay design}

The assay was designed to sequence all the coding sequences and splice donor and acceptance sites. In addition the promotor region and two intronic sequences were amplified to detect the mutations $1811+1.6 \mathrm{~kb} \mathrm{~A} \rightarrow \mathrm{G}$ and $3849+10 \mathrm{~kb} \mathrm{C} \rightarrow \mathrm{T}$. The scheme will detect 991 of the 1004 (98.7\%) described mutations as of August 2002.4 Table 1 is a listing of 13 known CF mutations not detected by this assay. All but two of these mutations involve large deletions of CFTR and would not be detectable by any sequencing assay.

Genomic DNA is purified from whole blood as described previously. ${ }^{7}$ Polymerase chain reaction (PCR) primer pairs were designed using the CFTR gene sequences in EMBL/Genbank (Accession Nos. M55106-M55131). Each PCR primer for the 32 separate PCR reactions contains either an M13 forward linker sequence or an M13 reverse linker sequence as appropriate to allow universal sequence reaction priming. Individual 
Table 1

CF mutations not detected by sequencing assay

\begin{tabular}{lr}
\hline Mutation & No. $^{a}$ \\
\hline$-816 \mathrm{C} \rightarrow \mathrm{T}$ & 60 \\
$-741 \mathrm{~T} \rightarrow \mathrm{G}$ & 59
\end{tabular}

CFTR50kbdel complex deletion involving exons 4-7 and 11-18

CFTR40kbdel deletion of exons 4-10

CFTR40kbdel deletion of exons 4-10

CFTRdele14a

CFTRdele14b-18

CFTRdele16-17b

CFTRdele17b18

CFTRdele21

CFTRdele19

2104insA+2109-2118del10

CF25kbdel

Data from Toronto Hospital for Sick Children's online database. ${ }^{4}$

${ }^{a}$ Identification number for Toronto Hospital for Sick Children's online database.

PCR reactions are performed in 96-well microtiter plates under the same conditions for each amplicon. Subsequently, the PCR products are purified with the Millipore Montage ${ }^{\mathrm{TM}}$ $\mathrm{PCR}_{96}$ Cleanup kit (Millipore, Bedford, MA) on a Beckman BioMek 2,000 biorobot. The purified PCR products are diluted to approximately $10 \mathrm{ng} / \mu \mathrm{L}$ and cycle sequencing reactions are performed with an ABI Prism Big Dye ${ }^{\mathrm{TM}}$ Terminator v3.0 cycle sequencing reaction kit (Applied Biosystems, Foster City, CA) according to the manufacturer's protocol. The DNA primers used for the sequencing reaction are M13 forward and reverse primers as appropriate. Big Dye ${ }^{\mathrm{TM}}$ Terminator reaction products are purified by the Millipore Montage ${ }^{\mathrm{TM}} \mathrm{Seq}_{96}$ Sequencing Reaction Cleanup kit on a biorobot and analyzed on an ABI Prism 3100 Genetic Analyzer. Sequences obtained were examined for the presence of mutations by using ABI SeqScape v1.1 software. Both strands of DNA were sequenced. In all, 10,132 bp are sequenced in both directions in 64 separate sequencing reactions. All PCR reactions, purifications, and cycle sequencing reactions are performed in 96-well microtiter plates using biorobots to avoid errors introduced by manual setups. Loading of samples onto the capillary sequencer is also automated. One plate is sufficient to perform the entire sequencing reaction for a single patient. Theoretically, if all reactions were successful, the entire sequence for a single patient could be obtained in 24-48 hours after receipt of blood. In practice, however, one or more reactions must be repeated because of frequent polymorphisms in intron 8 and 6 a and failed reactions. Single exon and two exon sequencing analysis to identify rare mutations have also proved invaluable in resolving ambiguities in carrier screening.

\section{RESULTS}

\section{Assay validation}

DNA from 17 patients with known mutations were made anonymous, coded, and submitted for analysis along with DNA from 3 wild-type controls. An additional patient with intriguing results in the carrier screening program was added after consent was obtained from her referring physician (see below). The laboratory was blinded to the identity of all samples and the expected mutations. The panel included compound heterozygous samples, homozygous mutant samples, and heterozygous samples. Table 2 is a listing of samples used for this validation. All mutations were correctly identified, and no mutations were identified in the normal controls.

In this validation series, several polymorphisms were identified in both the patients and controls used in the validation patients. These are summarized in Table 3 . In all, 15 previously described and 9 novel polymorphisms were detected. The 950 $\mathrm{G} \rightarrow \mathrm{A}$ and $1497 \mathrm{C} \rightarrow \mathrm{A}$ are clearly polymorphisms because they occur in exons and do not alter the encoded amino acid. The others occur in introns or untranslated regions. The $1811+1.6$ $\mathrm{kb}+16 \mathrm{~T} \rightarrow \mathrm{A}, 1899-136 \mathrm{~T} \rightarrow \mathrm{A}, 3850-71 \mathrm{C} \rightarrow \mathrm{T}$, and $1811+$ $229 \mathrm{~T} \rightarrow \mathrm{A}$ sequence variations occur in introns and at sufficient frequencies to be considered polymorphisms. The variants seen only once, 1342-64 delA, 106G $\rightarrow$ C, and 4095-100 A $\rightarrow$ G, could be CF mutations, but their occurrence in exons and untranslated regions makes it more likely that they represent polymorphisms. Only the 1342-64delA occurred in a known CF patient.

One extremely interesting patient, an asymptomatic pregnant women who was tested by her obstetrician for CF carrier status, was included in the validation series. The linear array strips yielded a result of homozygous for the rare mutation

Table 2

Mutant samples used for validation of sequencing assay

\begin{tabular}{l}
\hline Mutation expected \\
\hline wt/wt (3 patients) \\
delta F508/wt (2 patients) \\
R117H/wt (3 patients) \\
$2789+5 \mathrm{G} \rightarrow \mathrm{A} / 2789+5 \mathrm{G} \rightarrow \mathrm{A}$ (both parents confirmed carriers) \\
R117H/delta F508 (2 patients) \\
delta F508/I148T \\
delta F508/R1066C \\
delta F508/3848 + 10 kb C $\rightarrow \mathrm{T}$ \\
delta F508/G542X \\
R117H/I148T (2 patients) \\
2307 delA/N1303K \\
deltaF 508/711 + $1 \mathrm{G} \rightarrow \mathrm{T}$ \\
deltaF 508/1898 + $1 \mathrm{G} \rightarrow \mathrm{A}$ \\
G551D/N1303K
\end{tabular}


Table 3

List of polymorphisms detected in sequencing assay

\begin{tabular}{|c|c|c|c|}
\hline Polymorphisms & Location/description & $\begin{array}{c}\text { Patients } \\
\begin{array}{c}(n=19) \\
n(\%)\end{array}\end{array}$ & $\begin{array}{c}\text { Controls } \\
\begin{array}{c}(n=18) \\
n(\%)\end{array}\end{array}$ \\
\hline \multicolumn{4}{|l|}{ Known } \\
\hline $1001+11 \mathrm{C} / \mathrm{T}$ & +12 in intron $6 \mathrm{~b}$ & $9(47)$ & $3(17)$ \\
\hline TTGA repeat (5-7) & $5^{\prime}$ flanking region intron $6 a$ & $19(100)$ & $18(100)$ \\
\hline GT repeat $(8-10)$ & intron 8 & $19(100)$ & $18(100)$ \\
\hline $1898+152 \mathrm{~T} / \mathrm{A}$ & +152 intron 8 & $9(47)$ & $6(33)$ \\
\hline $1525-61 \mathrm{~A} / \mathrm{G}$ & -61 intron 9 & $10(52)$ & $7(39)$ \\
\hline $875+40 \mathrm{~A} / \mathrm{G}$ & +40 intron $6 \mathrm{a}$ & $2(11)$ & 0 \\
\hline $356 \mathrm{G} / \mathrm{A}$ & Arg or Gly at position 75 & 0 & $1(5.6)$ \\
\hline $2694 \mathrm{~T} / \mathrm{G}$ & exon 14 a, no amino acid change & $8(42)$ & $6(33)$ \\
\hline $3601-65 \mathrm{C} / \mathrm{A}$ & -65 intron 18 & $2(11)$ & 0 \\
\hline $4521 \mathrm{G} / \mathrm{A}$ & exon 24, no amino acid change & $4(21)$ & $1(5.6)$ \\
\hline $3272-93 \mathrm{~T} / \mathrm{C}$ & -93 intron $17 \mathrm{a}$ & $1(5.3)$ & $3(17)$ \\
\hline $1540 \mathrm{~A} / \mathrm{G}$ & met or val at position 470 & $2(11)$ & $4(22)$ \\
\hline $3030 \mathrm{G} / \mathrm{A}$ & exon 15 , no amino acid change & 0 & $1(5.6)$ \\
\hline $2691 \mathrm{~T} / \mathrm{C}$ & exon $14 \mathrm{a}$, no amino acid change & 0 & $1(5.6)$ \\
\hline $4002 \mathrm{~A} / \mathrm{G}$ & exon 20 , no amino acid change & $1(5.3)$ & 0 \\
\hline \multicolumn{4}{|l|}{ Novel } \\
\hline $3850-71 \mathrm{C} / \mathrm{T}$ & -71 intron 19 & $2(11)$ & $2(11)$ \\
\hline 1342-64 delA & -64 exon 8 & $1(5.3)$ & 0 \\
\hline $950 \mathrm{G} / \mathrm{A}$ & no amino acid change, exon $6 \mathrm{~b}$ & 0 & $1(5.6)$ \\
\hline $1811+229 \mathrm{~T} / \mathrm{A}$ & +229 intron 11 & $2(11)$ & $2(11)$ \\
\hline $106 \mathrm{G} / \mathrm{C}$ & $5^{\prime}$ untranslated region & $1(5.3)$ & 0 \\
\hline $1811+1.6 \mathrm{~kb}+16 \mathrm{~T} / \mathrm{A}$ & 16 bp downstream from mutation $1811+1.6 \mathrm{~kb} \mathrm{~A} \rightarrow \mathrm{G}$ & $9(47)$ & $3(17)$ \\
\hline 1899-136 T/A & -136 intron 12 & $8(42)$ & $3(16)$ \\
\hline $4096-100 \mathrm{~A} / \mathrm{G}$ & -100 intron 21 & 0 & $1(5.6)$ \\
\hline $1497 \mathrm{C} / \mathrm{A}$ & no amino acid change, exon 9 & 0 & $1(5.6)$ \\
\hline
\end{tabular}

$2789+5 \mathrm{G} \rightarrow \mathrm{A}$. The sequencing assay revealed this patient to be homozygous not only for this mutation but for 13 polymorphisms extending from exon 6a through exon 24 . The possibility of uniparental isodisomy or a large deletion was considered. Genotyping of the parents, however, revealed both parents to be heterozygous carriers of $2789+5 \mathrm{G} \rightarrow \mathrm{A}$. The parents are unrelated. These data indicate that this mutation probably occurred relatively recently in evolutionary terms.

Subsequently, we analyzed DNA from eight patients submitted by physicians on a research basis for sequencing. Seven of these patients were confirmed CF patients with positive sweat tests. One patient had multiple sweat tests giving conflicting results and bronchiectasis without malabsorption. Four of the seven confirmed CF patients had one CF mutation identified by extended panel screening and three had no mutations identified by either the ACMG or extended panel screening. Thus 4 of the 14 chromosomes in these 7 CF patients had identified mutations.Table 4 is a summary of extensive sequencing results for these patients. For the 10 unknown chromosomes, 7 described mutations were found and 2 novel missense mutations, D806G and F191L, were discovered. For one patient, one $\mathrm{CF}$ allele remained unidentified. Extensive sequencing of the "borderline" patient revealed no mutations, indicating that this patient may have another gene contributing to his symptoms and sweat test results.

\section{Resolving ambiguities in a carrier screening program}

CF testing for the ACMG panel uses the Roche CF Gold Linear Array strips. Irregularities such as unusually weak lines or missing lines can be present on the strip assay, and these ambiguities can be resolved by sequencing the appropriate amplicon. Table 5 is a listing of such cases and their resolution 
Table 4

Results of sequencing of patient samples

\begin{tabular}{|c|c|c|c|c|}
\hline \multirow[b]{2}{*}{ Description } & \multirow[b]{2}{*}{ Prior genotype } & \multicolumn{2}{|c|}{ Sequencing } & \multirow[b]{2}{*}{ Comment } \\
\hline & & Allele 1 & Allele 2 & \\
\hline Confirmed CF & wt/delta F508 & delta F508 & P205S & Known mutation \\
\hline \multirow[t]{2}{*}{ Confirmed CF } & $\mathrm{wt} / 3849+10 \mathrm{~kb}$ & $3849+10 \mathrm{~kb}$ & L1077P & Known mutation \\
\hline & $\mathrm{C} \rightarrow \mathrm{T}$ & $\mathrm{C} \rightarrow \mathrm{T}$ & & \\
\hline Confirmed CF & wt/delta F508 & delta F508 & R1066C & Known mutation \\
\hline Confirmed CF & wt/delta F508 & delta F508 & D806G & Novel missense \\
\hline Confirmed CF & wt/wt & 3154delG & 3154delG & Both parents confirmed carriers \\
\hline Confirmed CF & delta F508/wt & delta F508 & G1244E & Known mutation \\
\hline Confirmed CF & wt/wt & wt & F191L & Novel missense \\
\hline Borderline sweat test & wt/wt & wt & wt & \\
\hline
\end{tabular}

Table 5

Resolution of ambiguities on linear array assay using sequencing

\begin{tabular}{ll}
\hline Linear array result & \\
\hline Weak mutant A455E line & $1508 \mathrm{C} \rightarrow \mathrm{T}(\mathrm{S} 459 \mathrm{~F})$ polymorphism or novel mutation \\
Weak mutant A455E line & $1508 \mathrm{C} \rightarrow \mathrm{T}(\mathrm{S} 459 \mathrm{~F})$ polymorphism or novel mutation \\
Weak mutant A455E line & wt/1496 C $\rightarrow \mathrm{T}(\mathrm{A} 455 \mathrm{~V})$ polymorphism or novel mutation \\
Weak mutant A455E line & wt/1496 C $\rightarrow \mathrm{T}(\mathrm{A} 455 \mathrm{~V})$ polymorphism or novel mutation \\
Weak mutant A455E line & wt/1520 G $\rightarrow \mathrm{A}(\mathrm{G} 463 \mathrm{D})$ polymorphism or novel mutation \\
No A455E mutant or wt line & Homozygous $1499 \mathrm{~T} \rightarrow \mathrm{C}(\mathrm{V} 456 \mathrm{~A})$ polymorphism or novel mutation \\
No A455E mutant or wt line & Homozygous $1497 \mathrm{C} \rightarrow$ A polymorphism (no amino acid change) \\
Weak wt $1898+1 \mathrm{G} \rightarrow$ A line & wt/E587A novel missense mutation or polymorphism \\
Weak $1898+1 \mathrm{G} \rightarrow$ A line & wt/1898 $+1 \mathrm{G} \rightarrow \mathrm{C}-$ different mutation; G $\rightarrow$ C NOT G $\rightarrow$ A \\
\hline
\end{tabular}

using single amplicon sequencing. There were 9 instances of ambiguous results (approximately 1 per 10,000 assays). Seven of the nine involved the A455E mutation, and the remaining two involved the $1898+1 \mathrm{G} \rightarrow \mathrm{A}$ mutation. Weak positive lines for the A455E mutation were observed in five instances. Repeated analysis of the samples was performed beginning with DNA purification and gave identical weak positive results. Sequencing analysis revealed novel missense mutations or sequence variations in all five cases: two patients had $1508 \mathrm{C} \rightarrow \mathrm{T}$ (S459F) occurring four codons upstream from A455E, two had $1496 \mathrm{C} \rightarrow \mathrm{T}(\mathrm{A} 455 \mathrm{~V})$, and one had $1520 \mathrm{G} \rightarrow \mathrm{A}(\mathrm{G} 463 \mathrm{D})$. Whether these base changes represent novel CF mutations or polymorphism cannot be determined at this time, but the sequence analysis prevented the misdiagnosis of the patients as heterozygotes for A455E. In two cases there was no A455E signal in either the mutant or wild-type line. One of these patients was homozygous for a point mutation causing no change in amino acid $(1497 \mathrm{C} \rightarrow \mathrm{A})$. In the other patient, sequencing revealed homozygosity for a novel missense mutation V456A. As the patient was asymptomatic, we assume that this represents a polymorphism or mild CF mutation. Because of these un- usual results at the A455E site, we sequenced that exon for every patient previously found to be heterozygous for A455E. Ten patients were sequenced and the genotype A455E/wt was confirmed, indicating that the assay performance for this allele is acceptable when the expected intensity of the mutant band is observed. These data suggest that caution must be taken when weak A455E mutant bands are observed.

Two ambiguous results were obtained at the $1898+1 \mathrm{G} \rightarrow \mathrm{A}$ locus. A weak $1898+1 \mathrm{G} \rightarrow$ A mutant line was actually a different described CF mutation $1898+1 \mathrm{G} \rightarrow \mathrm{C}$. This distinction is critical for some assays such as the ABI Oligonucleotide Ligation Assay (OLA) kit, which would genotype this allele as wild-type. If this patient were to have a prenatal diagnosis and the sample was sent to a different laboratory, a misdiagnosis could ensue as the mutant $1898+1 \mathrm{G} \rightarrow \mathrm{C}$ would be interpreted as wild-type is some assays. Another patient with a weak $1898+1 \mathrm{G} \rightarrow \mathrm{A}$ line actually was heterozygous for a novel missense mutation E587A. Thus having the ability to perform single amplicon sequencing for all the mutations in the ACMG panel prevented nine misdiagnoses in just a 4 -month period of time. 


\section{DISCUSSION}

The ACMG recommended panel of CF mutations has rapidly become the standard of care for US carrier screening. Although a small number of laboratories offer larger panels than the ACMG recommendations, even these panels will fail to identify one or both CF mutations in many CF patients. For those patients and their families, extensive sequencing of CFTR has the potential to identify rare or novel CF mutations, for accurate carrier detection and prenatal diagnosis.

A sequence-based assay capable of detecting $98.7 \%$ of described mutations and also novel mutations throughout the coding sequence and splice junction regions of CFTR has been devised, validated, and implemented. The most direct advantage is for patients with CF when known CF mutations are discovered. Difficulties may arise when novel sequence changes are discovered whose significance may not be immediately apparent. In a patient with $\mathrm{CF}$, it may be reasonable to assume that any frame shift mutation, missense mutation, splice donor mutation, splice acceptor mutation, or nonsense mutation discovered is a true CF mutation. However, previous studies have shown that two such mutations, I148T and D1270N, are probably polymorphisms or low penetrance alleles. ${ }^{7}$ For those reasons, caution must be used when interpreting the presence of a rare or novel mutation found in the sequencing assay.

This becomes even more of an issue if the extensive sequencing assay is performed not on a CF patient, but on a relative of a CF patient or a patient with a negative family history. Spouses of patients with known CF mutations who have tested negative using the ACMG or an extended panel might reasonably request extensive sequencing, especially if they are of an ethnic group poorly detected by standard panels. If a novel sequence change is found in such a patient, genetic counseling must be provided to ensure the patient is aware that the sequence alteration may not, in fact, be a CF mutation. For that reason, we are soliciting specimens only from CF patients and their relatives.

An unexpected benefit of having the extensive sequencing, single exon sequencing, and two exon sequencing assays available has been the utility of using single exon sequencing to resolve ambiguities in our carrier screening program which currently uses the Roche CF Gold Linear Array. ${ }^{7}$ This is a hybridization-based assay and sometimes is affected by nearby polymorphisms or mutations occurring at the same nucleotide position the mutation the strips were designed to detect. Other hybridization-based assays such as Inogenetics will probably experience similar difficulties with some mutations. Sequencing the relevant exon has prevented a misdiagnosis in nine cases. Labeling a patient a carrier of the wrong mutation can have devastating effects. Many times a prenatal sample will be sent to a different laboratory from the laboratory that performed the carrier detection. This may be due to the differences in ordering patterns between the obstetrician-gynecologist ordering the initial carrier screen and the maternal-fetal medicine specialist performing the prenatal sampling or to insurance issues. Subtle differences such as the difference be- tween $1899+1 \mathrm{G} \rightarrow \mathrm{C}$ and $1898 \mathrm{G} \rightarrow$ A can lead to completely different interpretations in different assays. In fact, in our current assay an almost equal number of patients with positive mutant lines for the A455E mutation had sequence changes near the A455E locus as had true A455E mutations.

Another potential advantage of an extensive sequencing assay is the ability to effectively rule out CFTR mutations in patients with equivocal diagnoses for CF. It is highly unlikely that a patient with no detectable CF mutations by extensive sequence analysis would have CFTR-related CF. Further studies may determine that other genes can contribute to symptoms of $\mathrm{CF}$, or that in some cases CF may behave as a dominant disease.

Cutting et al. ${ }^{8}$ recently reported the cases of several individuals with CF symptoms and elevated sweat chloride levels who have nondetectable CFTR mutations by a similar extensive sequencing assay. They also reported two families in which linkage and haplotype analysis have definitively ruled out CFTR mutations as the cause of their clinical CF disease. ${ }^{8}$

Cutting et al. ${ }^{8}$ and we analyzed all exonic and bordering intronic sequences. However, our assay extended a minimum of $100 \mathrm{bp}$ into each intron at all intron-exon boundaries, whereas Cutting et al. sequenced 25 bp before each exon and 15 bp after each exon. Therefore, we were able to detect an additional 12 mutations: $711+34 \mathrm{~A} \rightarrow \mathrm{G}$; 876-10del8; 1249-29deIAT; 1249-27delTA; $1341+18 \mathrm{~A} \rightarrow \mathrm{G} ; 1811+18 \mathrm{G} \rightarrow \mathrm{A} ; 1898$ $+73 \mathrm{~T} \rightarrow \mathrm{G} ; 2752-26 \mathrm{~A} \rightarrow \mathrm{G} ; 3272-54 \operatorname{del} 704 ; 3272-26 \mathrm{~A} \rightarrow \mathrm{G}$; 4006-16del14; and 4096-28G $\rightarrow$ A. We also analyzed the promoter from $830 \mathrm{bp}$ upstream of the translation starting site as opposed to 290 bp by Cutting et al., ${ }^{8}$ thereby allowing us to detect the proposed regulatory mutations -471 delAGG and $-363 \mathrm{C} / \mathrm{T}$.

A previous study has shown that patients with chronic sinusitis have an increased frequency of heterozygosity for CF mutations. ${ }^{9}$ The observation that newborn screening programs using immunoreactive serum trypsinogen testing to identify CF heterozygotes demonstrates that even carriers have sufficient pancreatic duct blockage to cause an elevation of blood trypsinogen levels. ${ }^{10}$ Perhaps the CF patient in our study with only one identifiable CFTR mutation, a positive sweat test, and mild symptoms could be a symptomatic heterozygote. Other possibilities are that he has a second mutation undetected by this assay or he could have a mutation in another gene contributing to his abnormal sweat chloride measurement and mild symptoms. Continued use of the sequencing assay may shed light on this important issue.

Extensive sequencing of the CFTR gene will provide vital information for CF families when both CFTR mutations have not been identified by standard screening panels. The ability to selectively sequence single amplicons allows carrier detection and prenatal diagnoses for families with rare mutations not identified by standard screening programs. An unexpected benefit of single amplicon sequencing has been the ability to investigate ambiguous and unusual results obtained in the routine screening program, allowing us to prevent diagnostic errors. 


\section{Strom et al.}

Because the cost of performing extensive sequencing for the entire CFTR gene is more than $\$ 2000$, the test is designed only for CF patients, for relatives of known CF patients when the proband is unavailable for testing, and for clinicians to resolve diagnostic dilemmas in patients with equivocal clinical and/or laboratory findings. When a sequencing assay is ordered, a genetic counselor confirms the indication so that the inadvertent use of this test for carrier testing is prevented. Another reason to avoid extensive sequencing for carrier detection is the potential of finding novel missense mutations or rare mutations listed in the CF database that are not truly CF mutations. The discovery that the I148T and D1270N mutations are likely to be polymorphisms and not causative CF mutations points out the danger in assuming that all mutations listed in the CF database are causative mutations.

Extensive sequencing is not intended for use in carrier screening programs. When ambiguities are found in carrier screening, one does not need to sequence the entire CFTR. Single exon sequencing is appropriate in this instance at a comparable cost to the initial screen or an IVS8 reflex polymorphism test. Single exon sequencing can also be used for prenatal diagnosis and carrier detection for extended family members once a rare mutation has been identified.

A new use for single exon sequencing is the recent observation that I148T is only a CF chromosome in a haplotype that includes the 9T IVS8 polymorphism and 3199del6. ${ }^{11}$ In patients with I148T and 9T, single exon sequencing can determine whether 3199del6 is present. Other uses may become apparent with continued investigation of data derived from $\mathrm{CF}$ carrier screening programs.
The combination of extensive sequencing and single exon sequencing allows optimum flexibility for the laboratory and clinician to provide state-of-the-art information to their patients.

\section{References}

1. Rommens JM, Ianuzzi MC, Kerem B-S, Drumm ML, Melmer G, Dean M et al Identification of the cystic fibrosis gene: chromosome walking and jumping. Science 1989;245:1059-1065.

2. Riordan JR, Rommens JM, Kerem B-S, Alon N, Rozmahel R, Grzelczak Z et al Identification of the cystic fibrosis gene: cloning and characterization of complimentary DNA. Science 1989;245:1066-1073.

3. Kerem, B-S, Rommens JM, Buchanon JA, Markiewicz D, Cox TK, Chakravarti A et al. Identification of the cystic fibrosis gene: genetic analysis. Science 1989;245:10731080 .

4. Hospital for Sick Children, Toronto. Cystic Fibrosis Mutation Database. http:// www.genet.sickkids.on.ca/cftr/.

5. Grody WW, Cutting GR, Klinger KW, Richards CS, Watson M, Desnick RJ. Laboratory standards and guidelines for population-based cystic fibrosis carrier screening. Genet Med 2001;3:149-154

6. Palomacki GE, Haddow JE, Bradley LA, FitzSimmons SC. Updated assessment of cystic fibrosis mutation frequencies in non-Hispanic Caucasians. Genet Med 2002; 4:90-94.

7. Strom CM, Huang D, Buller A, Redman J, Crossley B, Anderson B et al. Cystic fibrosis screening using the College Panel: platform comparison and lessons learned from the first 20,000 samples. Genet Med 2002;4:289-296.

8. Groman J, Meyer ME, Wilmott RW, Zeitlin PL, Cutting GR. Variant cystic fibrosis phenotypes in the absence of CFTR mutations. N Engl J Med 2002;347:401-407.

9. Wang X, Moylan B, Leopold D, Kim J, Rubenstein RC, Togias A et al. Mutation in the gene responsible for cystic fibrosis and predisposition to chronic rhinosinusitis in the general population. JAMA 2000;284:1814-1817.

10. Corbetta C, Seia M, Bassotti A, Ambrosioni A, Guinta A, Padoan R. Screening for cystic fibrosis in newborn infants: results of a pilot programme based on a two tier protocol (IRT/DNA/IRT) in the Italian population. J Med Screen 2002;9:60-63.

11. Rohlfs EM, Zhou Z, Sugarman EA, Heim RA, Pace RG, Knowles MR et al. The I148T CFTR allele occurs on multiple haplotypes: a complex allele is associated with cystic fibrosis. Genet Med 2002;319-323. 